\title{
Diamond nitrogen-vacancy centers created by scanning focused helium ion beam and annealing
}

\author{
Z. Huang, ${ }^{1, a)}$ W.-D. Li, ${ }^{2, b)}$ C. Santori, ${ }^{1}$ V. M. Acosta,${ }^{1}$ A. Faraon, ${ }^{3}$ T. Ishikawa, ${ }^{4}$ W. Wu, ${ }^{5}$ \\ D. Winston, ${ }^{1}$ R. S. Williams, ${ }^{1}$ and R. G. Beausoleil ${ }^{1}$ \\ ${ }^{1}$ Hewlett-Packard Laboratories, Palo Alto, California 94304, USA \\ ${ }^{2}$ Department of Mechanical Engineering, The University of Hong Kong, Pokfulam, Hong Kong \\ ${ }^{3}$ Department of Applied Physics and Materials Science, California Institute of Technology, \\ Pasadena, California 91126, USA \\ ${ }^{4}$ Graduate School of Fundamental Science and Technology, Keio University, Yokohama, Japan \\ ${ }^{5}$ Department of Electrical Engineering, University of Southern California, Los Angeles, California 90089, USA
}

(Received 3 May 2013; accepted 12 August 2013; published online 23 August 2013)

\begin{abstract}
We demonstrate a method to create nitrogen-vacancy (NV) centers in diamond using focused helium ion microscopy. Near-surface NV centers can be created with spatial resolution below $0.6 \mu \mathrm{m}$. We studied the density, creation efficiency, and spectral linewidths at optical and microwave frequencies for NV centers produced using various helium ion implantation doses. The optical linewidths are narrower than those of similar nitrogen-vacancy centers produced using nitrogen ion implantation. (C) 2013 AIP Publishing LLC. [http://dx.doi.org/10.1063/1.4819339]
\end{abstract}

The negatively charged nitrogen-vacancy $\left(\mathrm{NV}^{-}\right)$center in diamond is promising as a solid-state qubit for quantum information processing (QIP) $)^{1-4}$ and for magnetic-field sensing. ${ }^{5,6}$ This is largely due to its unique combination of a long electronic spin coherence time and fast manipulation and readout at the single-spin level. ${ }^{7-9}$ For QIP applications, an important goal is to realize spin entanglement between an ensemble of distant NV centers using schemes based on optical interference. ${ }^{4,10}$ This has motivated many groups to develop optical microcavities with high quality factors and small mode volumes to enhance the photon emission rate into the desired spatial and spectral channel. ${ }^{11-17}$ Previously, most NV properties were studied in bulk diamond. However, near-surface NV centers are needed for efficient optical coupling to on-chip waveguides and cavities. For nanoscale magnetometry, near-surface NV centers will reduce the sensor-sample distance, thereby improving spatial resolution and sensitivity. ${ }^{18-21}$

The most commonly used technique to create NV centers near a surface is implantation of nitrogen ions followed by annealing at $900{ }^{\circ} \mathrm{C} .{ }^{22-24}$ Rabeau et al. reported a creation yield of $2.5 \%$ for the optically detectable $\mathrm{NV}^{-}$centers per implanted nitrogen $(\mathrm{N})$ ion. $^{22}$ Pezzagna et al. investigated a wide range of nitrogen implantation energies from $5 \mathrm{keV}$ to $18 \mathrm{MeV}$ and gave the dependence of the NV yield on the ion energies. $^{25}$ Naydenov et al. determined that co-implantation of carbon and nitrogen ions increases the $\mathrm{NV}^{-}$conversion efficiency to up to $50 \% .{ }^{24}$ Methods to implant nitrogen at the desired position using a focused nitrogen ion beam were also reported by Meijier et al. ${ }^{23}$ and Toyli et al. ${ }^{26}$ Besides introducing single vacancies, it is suspected that heavy ion bombardment introduces undesirable defects and impurities that might create charge traps or paramagnetic centers. Experiments typically find that NV centers formed from such a process suffer from increased optical spectral

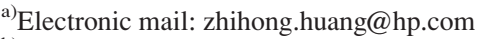

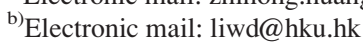

diffusion and degraded spin coherence properties. ${ }^{27,28}$ As an alternative, electron irradiation and annealing can be used to convert existing substitutional nitrogen into NV centers. ${ }^{29}$ Experiments have shown that the spectral properties of NV centers produced by electron irradiation are similar to the properties of as-grown NV centers. However, for the large energies required to create damage, the correspondingly large penetration depth creates NVs throughout the bulk diamond.

Here, we investigate the use of a light-weight ion, helium, to create vacancies that can combine with existing nitrogen upon annealing. By using a light-weight ion, it is hoped that implantation damage will be less dense and less clustered so that NV centers produced following annealing will have properties similar to those produced by electron irradiation. Furthermore, the use of a helium ion microscope with sub-nanometer spot size allows controlled placement of NV centers as needed for the applications discussed above.

SRIM simulations indicate that for helium ion implantation into bulk diamond, lattice vacancies will be created to a maximum depth of $100-200 \mathrm{~nm}$ for implantation energies ranging from 15 to $35 \mathrm{keV}$, with lateral straggling over similar dimensions. Previously, the helium-ion microscope has proved its capability for high-resolution imaging $(0.24 \mathrm{~nm}$ resolution) and lithography (dense lines with a half-pitch of $4 \mathrm{~nm}$ were demonstrated). ${ }^{30}$ In addition to the benefit of nanometer spatial resolution, the microscope also has the capability of obtaining a large range of ion fluences without the need for repeated implantation. In this letter, we demonstrate application of the focused helium ion microscope to create NV centers in diamond.

Two types of high-pressure, high-temperature (HPHT) synthesized diamond plates with nitrogen concentration differing by at least two orders of magnitude were studied (Sumitomo $[N]<100 \mathrm{ppm}(\mathrm{S} 1)$ and $[N]<1 \mathrm{ppm}$ (S2)). Both samples were implanted at $30 \mathrm{kV}$ acceleration voltage and subsequently annealed at $900{ }^{\circ} \mathrm{C}$ for $2 \mathrm{~h}$, in $\mathrm{H}_{2}$ Ar forming gas, for vacancy diffusion, followed by another annealing at 
$450{ }^{\circ} \mathrm{C}$ in $\mathrm{O}_{2}$ to increase the $\mathrm{NV}^{-}$conversion. ${ }^{27}$ Finally, an $\mathrm{O}_{2}$ descum in a reactive ion etcher was used to remove fluorescent organic molecules on the surface. Confocal photoluminescence (PL) microscopy was performed on the samples using laser excitation at $532 \mathrm{~nm}$. The PL from $\mathrm{NV}^{-}$phonon sidebands $650-800 \mathrm{~nm}$ was collected through a 0.6 numerical aperture microscope objective or an oil-immersion objective $(\mathrm{NA}=1.3)$. High-resolution emission spectroscopy was performed at both room temperature and $7 \mathrm{~K}$.

Fig. 1(a) shows a confocal PL image of a helium-ionimplanted region on the type-Ib plate (S1). A $10 \times 10$ array of $5 \times 5 \mu \mathrm{m}^{2}$ squares was implanted in the sample with ion doses increasing geometrically from $10^{8}-10^{14} \mathrm{~cm}^{-2}$. In the PL scan image, the colors indicate the photon count rates for $\mathrm{NV}$ phonon sideband emission. The dose from stray ions is estimated less than $10^{9} \mathrm{~cm}^{-2}$, as the patterns at that dose rang are still resolvable. Fig. 1(b) shows typical PL for the created NV centers at room and cryogenic temperature with clear $\mathrm{NV}^{-}$zero-phonon line (ZPL) signatures at $637 \mathrm{~nm}$. The image in Fig. 1(c) is obtained with one of the scan axes in the vertical direction, indicating that NV centers are positioned within $5 \mu \mathrm{m}$ of the surface, limited by the optical resolution. In comparison, electron irradiation causes a relatively even distribution of NV centers throughout a diamond plate. ${ }^{5}$ We also implanted dot arrays to examine the lateral resolution. Each dot is implanted in a single pulse, and its size is ideally limited by helium ion lateral straggle and vacancy diffusion. A PL scan image of a $0.6 \mu \mathrm{m}$ spacing dot array is shown in Fig. 1(d).

The absolute sheet density of the NV centers is a figure of merit of interest for magnetometry applications. We estimated this quantity by comparing the measured PL intensities with those of single NV centers that can be resolved in high-purity samples, taking into account the collection area of the setup. Subsequently, the volume density can be estimated by dividing the sheet density by the average implantation depth $(d z)$ simulated by SRIM, provided that $d z$ is much
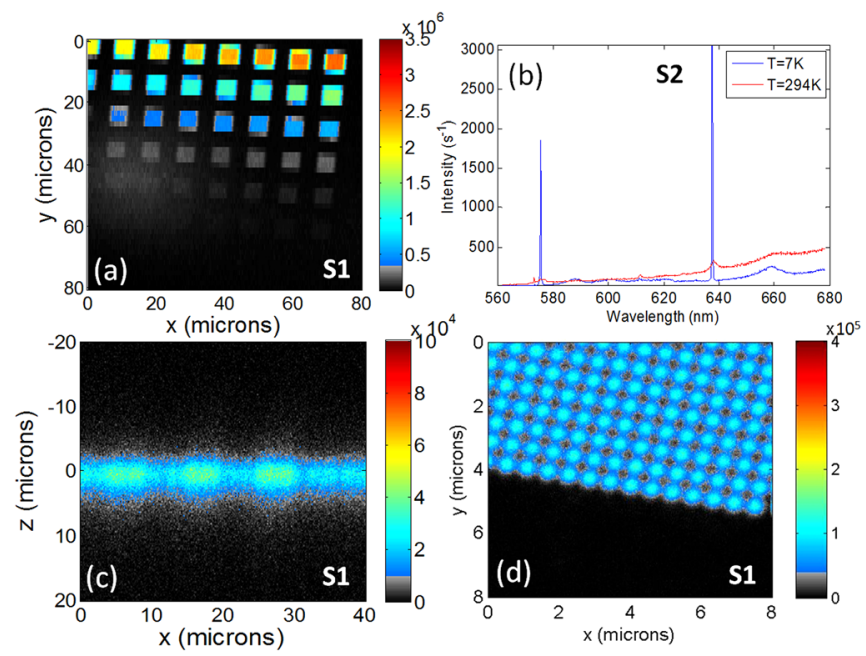

FIG. 1. (a) $5 \times 5 \mu \mathrm{m}^{2}$ dose array created by a focused helium ion beam for sample S1; false color represents intensity. (b) Spectra of sample S2 at room temperature and 7K. (c) Confocal xz-scan. The light is focused on the sample surface at $z=0$ and $z>0$ denotes focal positions inside the sample. The $\mathrm{z}$-axis is scaled by the diamond refractive index $(n=2.4)$. (d) PL image of $0.6 \mu \mathrm{m}$ spacing dot array. smaller than the depth of focus of the optical setup. This method may overestimate the volume density, since vacancy diffusion during annealing may increase the effective $d z$.

Figure 2(a) shows the estimated NV sheet densities for sample S1 and S2. Both samples show a linear increase of $\mathrm{NV}$ density with implantation dose, except for a signature of saturation at the higher dose: $10^{14} \mathrm{~cm}^{-2}$ for the type-Ib diamond (S1) and $10^{11} \mathrm{~cm}^{-2}$ for the type-IIa sample (S2), respectively. In estimating the NV conversion efficiency, we assume that the depth is given by the calculated vacancy positions given by SRIM and the maximum specified nitrogen concentrations of $100 \mathrm{ppm}$ and $1 \mathrm{ppm}$, respectively. With doses in the $10^{11}-10^{12} \mathrm{~cm}^{-2}$ range, the NV conversion efficiency of the type Ib material (S1) is two orders of magnitude lower than that of the type IIa sample (S2). For $\mathrm{S} 1$, in the presumed saturation region, the estimated NV conversion efficiency is $10 \%$. With $30 \mathrm{keV}$ of implantation energy, the SRIM simulation indicates that every helium ion creates 22 vacancies throughout the depth it penetrates. Thus for a dose of $10^{14} \mathrm{~cm}^{-2}$ at $d z=200 \mathrm{~nm}$, we expect a vacancy density of $10^{20} \mathrm{~cm}^{-3}$, which is greater than the expected nitrogen density ( $100 \mathrm{ppm}$ is equivalent to $\left.1.76 \times 10^{19} \mathrm{~cm}^{-3}\right)$. The highest conversion efficiencies estimated here are similar to those obtained through pure nitrogen implantation at a similar energy range. ${ }^{22,28}$ Higher conversion efficiencies, up to $50 \%$, have only been obtained with $\mathrm{MeV}$ nitrogen implantation energies ${ }^{25}$ or with nitrogen and carbon co-implantation. ${ }^{24}$

Next, we discuss the optical spectral linewidths for NV centers created using this method. Since sample (S1) has broad linewidths $(0.2 \mathrm{~nm})$ even for native NV centers, here we focus on sample (S2), in which the native NVinhomogeneous linewidths of the ZPL are as narrow as $0.04 \mathrm{~nm}$. Figure 2(b) shows the ZPL linewidth vs. helium implantation dose over a range of $532 \mathrm{~nm}$ excitation powers. Except for a few outliers, we observe a trend of decreasing $\mathrm{NV}^{-}$ensemble ZPL linewidth with reduced implantation dose. Below a dose of $10^{11} \mathrm{~cm}^{-2}$, the linewidths are similar to those of native $\mathrm{NV}$ ensembles on the same sample,

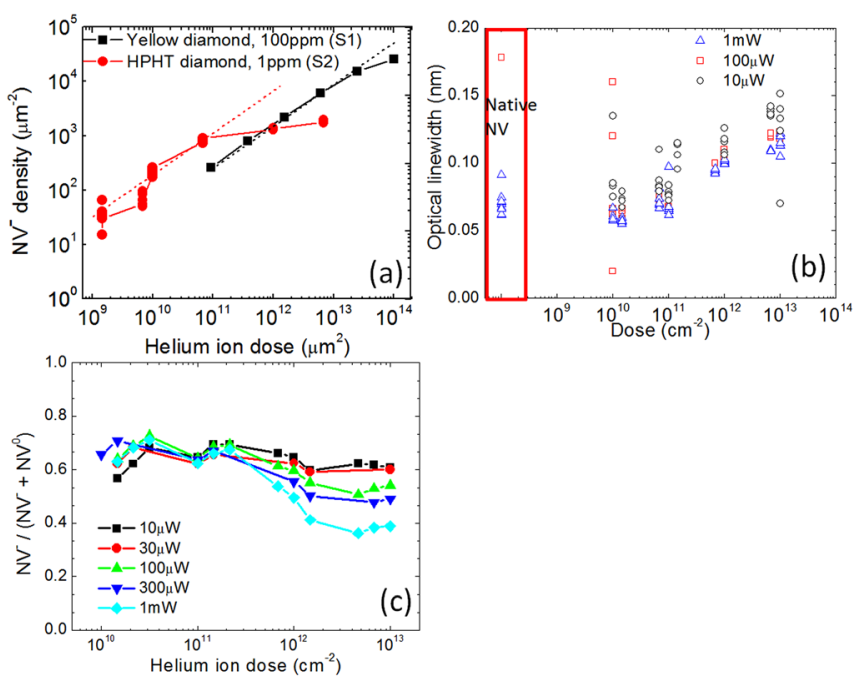

FIG. 2. (a) Calculated $\mathrm{NV}^{-}$density vs. dose for samples $\mathrm{S} 1$ and $\mathrm{S} 2$. NV density is calibrated from a single $\mathrm{NV}^{-}$intensity. (b) $\mathrm{NV}^{-}$ZPL linewidth versus dose for sample $\mathrm{S} 2$ at different optical powers. (c) $\mathrm{NV}^{-} / \mathrm{NV}^{0}$ ratio for sample $\mathrm{S} 2$ versus implantation dose. 

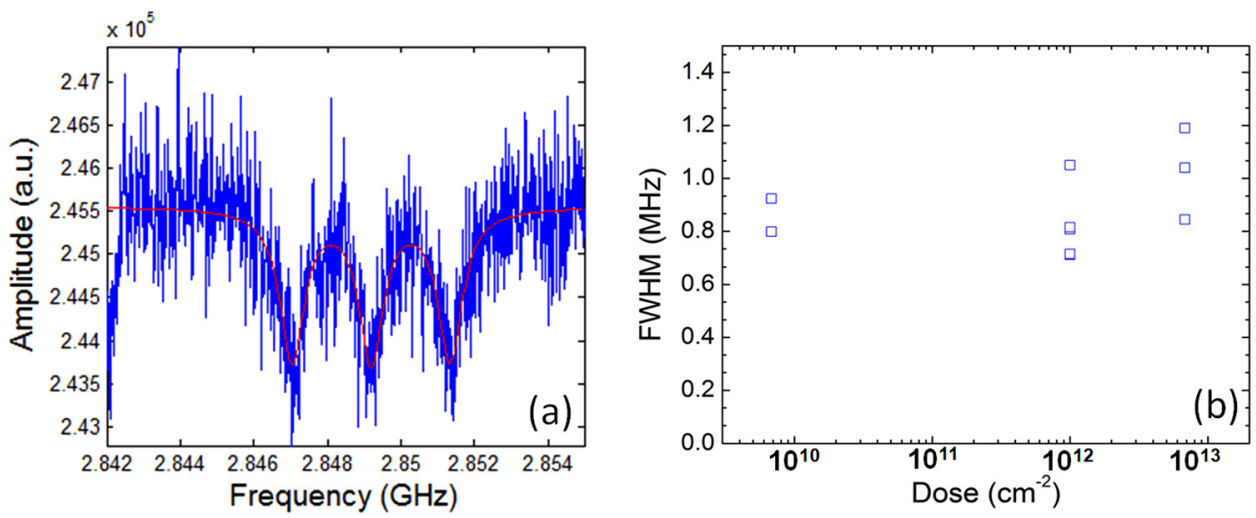

FIG. 3. (a) cw-ODMR scan for sample S2. (b) Extrapolated ODMR linewidth vs dose for sample S2.
$25 \mathrm{GHz}$ full-width-at-half-maximum (FWHM). As a comparison, nitrogen implantation at $10 \mathrm{keV}$ and $10^{10} \mathrm{~cm}^{-2}$ dose into type-IIa electronic grade material typically generates $\gtrsim 100 \mathrm{GHz}$ inhomogeneous linewidths.

Although NV centers are created by combining a nitrogen defect with a vacancy in diamond, only the negatively charged NV centers have the optical and spin properties of interest for quantum information and magnetic sensing. The likelihood of an NV center to be in a particular charge state is affected by the concentration of nearby electronic donors and acceptors. To increase the $\mathrm{NV}^{-}$fraction, both sample $\mathrm{S} 1$ and $\mathrm{S} 2$ were annealed in $\mathrm{O}_{2}$ to form an oxygen-terminated surface. $^{27}$ For sample S1, we calculated the integrated ZPL intensity ratio between $\mathrm{NV}^{-}$and the sum of the $\mathrm{NV}^{-}$and $\mathrm{NV}^{0}$ to be consistently greater than $60 \%$ at $50 \mu \mathrm{W}$ optical power. For sample $\mathrm{S} 2$, the $\mathrm{NV}^{-}$ratios were measured at various optical powers and plotted in Figure 2(c). At a high optical excitation power, the drop of the $\mathrm{NV}^{-}$fraction in high implantation dose regions suggests the occurrence of electron photo-ionization in the NV center. At these powers, we observe a dependence of the dynamic NV center photochromic behavior on helium ion dose. ${ }^{31,32}$

Finally, to investigate the spin properties, we measured continuous-wave optically detected magnetic resonance (cwODMR) spectra for $\mathrm{S} 2$ at room temperature. In the experiment, the microwave and optical excitations were at constant powers, and the fluorescence intensity into the phonon sidebands was measured as a function of microwave frequency. Close examination of a single $m_{s}=0$ and $m_{s}= \pm 1$ transition reveals hyperfine structure associated with the nitrogen nuclear spin $(I=1)$ in the NV center. Since the electron spin resonance transitions preserve the nuclear spin state, three allowed transitions are separated by $A_{\|}=2.2 \mathrm{MHz}$ as shown in Figure 3(a). High microwave power was initially used to locate the optimal resonance frequency, and the final ODMR spectrum was taken at a low microwave and optical power to avoid power broadening. In Figure 3(b), the fitted linewidth is plotted as a function of dose, showing that the ODMR linewidth remains in the range of $0.8-1 \mathrm{MHz}$ for doses from $10^{10}-10^{13} \mathrm{~cm}^{-2}$. The time constant $T_{2}^{*}$, a parameter indicating DC magnetic field sensitivity and quantum information storage time in the absence of refocusing techniques, is estimated to be $400 \mathrm{~ns}$ based on the measured ODMR linewidth. ${ }^{5}$

In this work, we demonstrated a method to create nitrogen vacancy centers using a focused helium ion microscope. We formed near-surface NV centers with $0.6 \mu \mathrm{m}$ spatial resolution and obtained better ensemble optical properties than are typically seen for nitrogen implantation for similar implantation depths and annealing procedures. This technique is useful for controlled placement of NV centers within optical structures or near a diamond surface, as needed for quantum information and magnetic sensing applications, respectively.

This manuscript is based on work supported by the Defense Advanced Research Projects Agency (Award No. HR0011-09-1-0006) and the Regents of the University of California. W.-D. Li was supported in part by the Small Project Funding (201209176202) from the University of Hong Kong. We thank Professor Kai-Mei Fu at the University of Washington for helpful discussions.

${ }^{1}$ A. Gruber, A. Dräbenstedt, C. Tietz, L. Fleury, J. Wrachtrup, and C. von Borczyskowski, Science 276, 2012 (1997).

${ }^{2}$ F. Jelezko, T. Gaebel, I. Popa, M. Domhan, A. Gruber, and J. Wrachtrup, Phys. Rev. Lett. 93, 130501 (2004).

${ }^{3}$ F. Jelezko, T. Gaebel, I. Popa, A. Gruber, and J. Wrachtrup, Phys. Rev. Lett. 92, 076401 (2004).

${ }^{4}$ L. Childress, J. M. Taylor, A. S. Sorensen, and M. D. Lukin, Phys. Rev. Lett. 96, 70504 (2006).

${ }^{5}$ V. M. Acosta, E. Bauch, M. P. Ledbetter, C. Santori, K.-M. C. Fu, P. E. Barclay, R. G. Beausoleil, H. Linget, J. F. Roch, F. Treussart, S. Chemerisov, W. Gawlik, and D. Budker, Phys. Rev. B 80, 115202 (2009).

${ }^{6}$ J. M. Taylor, P. Cappellaro, L. Childress, L. Jiang, D. Budker, P. R. Hemmer, A. Yacoby, R. Walsworth, and M. D. Lukin, Nat. Phys. 4, 810 (2008).

${ }^{7}$ L. Robledo, L. Childress, H. Bernien, B. Hensen, P. F. A. Alkemade, and R. Hanson, Nature 477, 574 (2011).

${ }^{8}$ B. B. Buckley, G. D. Fuchs, L. C. Bassett, and D. D. Awschalom, Science (New York, N.Y.) 330, 1212 (2010).

${ }^{9}$ G. Balasubramanian, I. Chan, R. Kolesov, M. Al-Hmoud, J. Tisler, C. Shin, C. Kim, A. Wojcik, P. R. Hemmer, A. Krueger et al., Nature 455, 648 (2008).

${ }^{10}$ H. Bernien, B. Hensen, W. Pfaff, G. Koolstra, M. Blok, L. Robledo, T. Taminiau, M. Markham, D. Twitchen, L. Childress et al., Nature 497, 86 (2013).

${ }^{11}$ C. Kreuzer, J. Riedrich-Möller, E. Neu, and C. Becher, Opt. Express 16, 1632 (2008).

${ }^{12}$ Y.-S. Park, A. K. Cook, and H. Wang, Nano Lett. 6, 2075 (2006).

${ }^{13}$ A. Faraon, P. E. Barclay, C. Santori, K. -m. C. Fu, and R. G. Beausoleil, Nat. Photonics 5, 301 (2011).

${ }^{14}$ A. Faraon, C. Santori, Z. Huang, V. M. Acosta, and R. G. Beausoleil, Phys. Rev. Lett. 109, 33604 (2012).

${ }^{15}$ I. Aharonovich, A. D. Greentree, and S. Prawer, Nat. Photonics 5, 397 (2011).

${ }^{16}$ M. Barth, N. Nüsse, B. Löchel, and O. Benson, Opt. Lett. 34, 1108 (2009).

${ }^{17}$ C. F. Wang, R. Hanson, D. D. Awschalom, E. L. Hu, T. Feygelson, J. Yang, and J. E. Butler, Appl. Phys. Lett. 91, 201112 (2007).

${ }^{18}$ T. Ishikawa, K.-M. C. Fu, C. Santori, V. M. Acosta, R. G. Beausoleil, H. Watanabe, S. Shikata, and K. M. Itoh, Nano Lett. 12, 2083 (2012). 
${ }^{19}$ K. Ohno, F. J. Heremans, L. C. Bassett, B. A. Myers, D. M. Toyli, A. C. B. Jayich, C. J. Palmstrom, and D. D. Awschalom, Appl. Phys. Lett. 101, 082413 (2012).

${ }^{20}$ P. Maletinsky, S. Hong, M. S. Grinolds, B. Hausmann, M. D. Lukin, R. L. Walsworth, M. Loncar, and A. Yacoby, Nat. Nanotechnol. 7, 320 (2012).

${ }^{21}$ H. Mamin, M. Sherwood, and D. Rugar, Phys. Rev. B 86, 195422 (2012).

${ }^{22}$ J. R. Rabeau, P. Reichart, G. Tamanyan, D. N. Jamieson, S. Prawer, F. Jelezko, T. Gaebel, I. Popa, M. Domhan, and J. Wrachtrup, Appl. Phys. Lett. 88, 23113 (2006).

${ }^{23}$ J. Meijer, B. Burchard, M. Domhan, C. Wittmann, T. Gaebel, I. Popa, F. Jelezko, and J. Wrachtrup, Appl. Phys. Lett. 87, 261909 (2005).

${ }^{24}$ B. Naydenov, V. Richter, J. Beck, M. Steiner, P. Neumann, G. Balasubramanian, J. Achard, F. Jelezko, J. Wrachtrup, and R. Kalish, Appl. Phys. Lett. 96, 163108 (2010).
${ }^{25}$ S. Pezzagna, B. Naydenov, F. Jelezko, J. Wrachtrup, and J. Meijer, New J. Phys. 12, 065017 (2010).

${ }^{26}$ D. M. Toyli, C. D. Weis, G. D. Fuchs, T. Schenkel, and D. D. Awschalom, Nano Lett. 10, 3168 (2010).

${ }^{27}$ K. -M. C. Fu, C. Santori, P. E. Barclay, and R. G. Beausoleil, Appl. Phys. Lett. 96, 121907 (2010).

${ }^{28}$ I. Aharonovich, C. Santori, B. Fairchild, J. Orwa, K. Ganesan, K. Fu, R. Beausoleil, A. Greentree, and S. Prawer, J. Appl. Phys. 106, 124904 (2009).

${ }^{29}$ L. C. Bassett, F. J. Heremans, C. G. Yale, B. B. Buckley, and D. D. Awschalom, Phys. Rev. Lett. 107, 266403 (2011).

${ }^{30}$ W.-D. Li, W. Wu, and R. S. Williams, J. Vac. Sci. Technol. B 30, 06 F304 (2012).

${ }^{31}$ K. Iakoubovskii, Diamond Relat. Mater. 9, 861 (2000).

${ }^{32}$ N. Manson and J. Harrison, Diamond Relat. Mater. 14, 1705 (2005). 